\title{
GASTRO-ESOPHAGEAL REFLUX DISEASE IN DISGUISE OF
}

\section{OTHERS PROBLEMS}

\author{
Prof. AHM Rowshon ${ }^{1}$. Dr. Mohammed Masudur Rahman ${ }^{2}$
}

Gastroesophageal reflux disease (GERD) is common. At least 20 percent of respondents in one survey reported heartburn weekly. Of this, 10 percent have endoscopic evidence of esophagitis, according to another estimate. It is estimated that GERD accounts for more than 1.8 million outpatient office visits a year in the USA. It accounts for nearly $\$ 10$ billion in annual costs for diagnosis and treatment. It also undermines the quality of life more than arthritis, myocardial infarction, heart failure and hypertension.

It is common knowledge that GERD causes heartburn, frequent burping and belching, water brash, chest pain and swallowing difficulties. What is not known to most patients is that GERD could be associated with atypical symptoms and signs at times and could be missed easily if not actively looked for.

For example, GERD could be associated with recurrent sinus and ear infections, loss of enamel from teeth, recurrent sore throats, hoarseness, laryngitis, chronic cough, asthma, vocal cord dysfunction and recurrent pneumonia from macro- or microaspiration of stomach contents into lungs and scarring of lungs. It could cause swallowing difficulties, poor weight gain and gross or occult loss of blood in the stool, vomiting of blood, anemia and cancer of the esophagus.

GERD could be confused with a heart attack because of the severe chest pain it causes at times.

Esophagus and Larynx both tubes also have common nerve supply from the brain - the 10th cranial nerve called the vagus nerve supplies both. Therefore, it is not surprising that acid irritation of the esophagus causes neurologically arbitrated reflex constriction of the bronchus and causes asthma and chronic cough. It is estimated that nearly 20 percent of people who cough for more than eight weeks do so because of GERD. The cough gets better once GERD gets treated.

In one pediatric study, nearly 90 percent of children's asthma got significantly better following diagnosis and treatment of GERD for one year with medications.

Asthma gets worse for several reasons during the night. Gastroesophageal reflux is one of them. GERD should be suspected in patients with asthma whose symptoms typically flare up suddenly during night and who cough or choke during or following meals.
Chronic irritation of vocal cords from acid exposure also makes the vocal cords more irritable. This results in paradoxical vocal cord spasm and sudden closure of airway resulting in sudden temporary loss of voice and difficulty breathing at times. This is known as Vocal Cord Dysfunction. The VCD should be suspected in patients with asthma whose asthma gets suddenly worse and is associated with laryngeal symptoms such as hoarseness and temporary and transient loss of voice.

Proper diagnosis and treatment of GERD helps patients with VCD and asthma.

GERD should be suspected in children who fail to gain weight adequately and who vomit easily following crying or coughing. The weight loss occurs because of loss of calories from vomiting and poor intake of food because of esophagitis. The gastroesophageal reflux is more common in infants who are born prematurely and during the first year of life. As the child assumes a more erect posture, starts eating more solid food and as the maturity of the valve that guards the lower end of esophagus improves, the reflux gets better.

A word of caution is needed here, however. On rare occasions, children presenting with symptoms resembling GERD may have a condition known as Eosinophilic Esophagitis. The cause for this condition is not known. Food allergy is suspected in some. This condition should be suspected in children who seemingly have GERD and who do not improve even after acceptable treatment of GERD. Endoscopy and biopsy are needed to prove the diagnosis.

So, if any one has one of the aforementioned conditions, the consulting doctor should pay extra attention to exclude GERD.

1. Dr. A H M Rowshon Professor of Gastroenterology Shaheed Suhrawardy Medical College. Dhaka

1. Dr. Mohammed Masudur Rahman Assistant Professor of Gastroenterology Shaheed Suhrawardy Medical College. Dhaka 